BULL. AUSTIRAL. MATH. SOC.

VoL. 30 (1984), 307-314.

\title{
LINEAR SYSTEMS OF NONOSCILLATURY DIFFERENTIAL EQUATIONS WITH DELAYED ARGUMENTS
}

\author{
K. Gopalsamy
}

Sufficient conditions are obtained for a not necessarily scalar system of the form

$$
\frac{d^{n} x(t)}{d t^{n}}+(-1)^{n-1} A(t) X(t-\tau(t))=0
$$

to be nonoscillatory.

\section{Introduction}

The purpose of this paper is to obtain a set of sufficient conditions under which the $n$-th order nonautonomous differential difference equations of the form

$$
\frac{d^{n} x(t)}{d t^{n}}+(-1)^{n-1} \sum_{j=1}^{m} a_{j}(t) x\left(t-\tau_{j}(t)\right)=0
$$

where $n$ and $m$ are positive integers $(n \geqq 1, m \geqq 1)$ will have a nonoscillatory solution and subsequently generalise this result for nonscalar systems. Although the literature concerned with oscillation of differential equations with and without deviating arguments is quite extensive (see Kartsatos [2]) published work dealing with the existence of nonoscillatory solutions of differential difference equations is rather scarce. Using the characteristic equation associated with an autonomous majorant of (1.1) we will obtain first sufficient conditions for (1.1) to have nonoscillatory solutions.

As it is customary we will say that (1.1) is oscillatory if and

Received 22 May 1984.

Copyright Clearance Centre, Inc. Serial-fee code: v004-9727/84 $\$ A 2.00+0.00$. 
only if all solutions of (1.1) defined on an interval of the form $[\alpha, \infty)$ for some real constant $\alpha$ have zeros on every interval of the form $[\beta, \infty)$ for $\beta \geqq \alpha$. (1.1) will be called nonoscillatory if (1.1) has at least one solution which has no zeros on an interval of the form $[\gamma, \infty)$ for some $\gamma \geq \alpha$.

\section{2. lionoscillatory scalar systerns}

We will examine (1.1) under the following assumptions: $\left(H_{1}\right) a_{j}, \tau_{j}(j=1,2, \ldots, m)$ are bounded continuous functions defined on $[0, \infty)$ such that the following hold;

(2.1)

$$
\left.\begin{array}{l}
0 \leqq a_{j}(t) \leqq a_{j}^{*} \\
0 \leqq \tau_{j}(t) \leqq \tau_{j}^{*} \\
0 \leqq t-\tau_{j}(t)
\end{array}\right\} j=1,2, \ldots, n ; t \geqq 0
$$

(2.2) $\left(H_{2}\right) \quad\left(\sum_{j=1}^{n} a_{j}^{*}\right) \tau^{n} e^{n} / n^{n} \leqq 1 ; \tau=\max \left(\tau_{1}^{*}, \tau_{2}^{*}, \ldots, \tau_{n}^{*}\right)$

Let us now consider the autonomous "majorant" of (1.1) in the form

$$
\frac{d^{n} y(t)}{d t^{n}}+(-1)^{n-1} \sum_{j=1}^{m} a_{j}^{*} y\left(t-\tau_{j}^{*}\right)=0 \text {. }
$$

The characteristic equation associated with (2.3) is given by

$$
\lambda^{n}=(-1)^{n} \sum_{j=1}^{m} a_{j}^{*} \exp \left[-\lambda \tau_{j}^{*}\right]
$$

which with $\lambda=-\mu$ reads

$$
\mu^{n}=\sum_{j=1}^{m} a_{j}^{*} \exp \left[\mu \tau_{j}\right]
$$

We will show that when (2.2) holds, (2.5) has a real positive root.

Suppose first $m=1$ and consider $g:[0, \infty) \rightarrow(-\infty, \infty)$ defined by

$$
g(\mu)=\mu^{n}-a_{1}^{*} \exp \left[\mu \tau_{1}^{*}\right] \text {. }
$$

It will follow from

$$
g(0)=-a_{1}^{*}<0
$$

and 
Differential equations with aelayed arguments

$$
\begin{aligned}
g\left(\frac{n}{\tau_{1}^{*}}\right) & =\left(n / \tau_{1}^{*}\right)^{n}-a_{1}^{*} \exp \left[\tau_{1}^{*}\left(n / \tau_{1}^{*}\right)\right] \\
& =\left(n / \tau_{1}^{*}\right)^{n}\left[1-a_{1}^{*} e^{n}\left(\tau_{1}^{*}\right)^{n} / n^{n}\right] \\
& \geqq 0
\end{aligned}
$$

that $g$ has a zero on $\left[0, n / \tau_{1}^{*}\right]$. The general case with $m \geqq 2$ is treated as follows. We rewrite (2.5) in the form

$$
\begin{aligned}
\sigma & =\sum_{j=1}^{m} a_{j}^{*} \exp \left[\tau_{j}^{*}(\sigma)^{1 / n}\right] \\
& =h(\sigma) \quad \text { (say). }
\end{aligned}
$$

Since

$$
\frac{d h(\sigma)}{d \sigma}=\sum_{j=1}^{m} a_{j}^{*} \exp \left[\tau_{j}^{*}(\sigma)^{1 / n}\right]\left(\tau_{j}^{*} / n\right)(\sigma)^{(1-n) / n},
$$

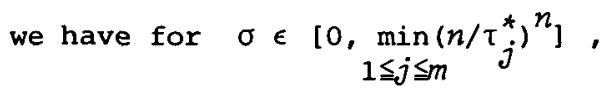

$$
\begin{aligned}
\frac{d h(\sigma)}{d \sigma} & <\left[\sum_{j=1}^{m} a_{j}^{*}\left(\tau_{j}^{*}\right)^{n}\right] e^{n} / n^{n} \\
& \leq\left\{\left[\sum_{j=1}^{m} a_{j}^{*}\left(\tau_{j}^{*}\right)^{n}\right] e^{\left.n / n^{n}\right\}-\varepsilon}\right. \\
& \leq 1-\varepsilon \text { for } \operatorname{some} \varepsilon, 0<\varepsilon<1 \\
& \leq 0<\sigma<\sigma^{*}=\underset{i \leq j \leq m}{\min \left(n / \tau_{j}^{*}\right)^{n} .}
\end{aligned}
$$

One can now show that a sequence $\left\{\sigma_{k}\right\}, k=0,1,2, \ldots$ defined by

$$
\sigma_{k+1}=h\left(\sigma_{k}\right), k=0,1,2, \ldots
$$

for an arbitrarily chosen $\sigma_{0} \epsilon\left(0, \sigma^{*}\right)$ will converge to a limit say $\sigma_{0}>0$ which is a root of $(2.6)$. Corresponding to this $\sigma_{0}$ we have a negative root of the characteristic equation given by $\lambda=-\left(\sigma_{0}\right)^{1 / n}$. We are now ready to formulate the following:

THEOREM 2.1. Suppose the coefficients $a_{j}$ and the delays $\tau_{j}(j=1,2, \ldots, n)$ satisfy the hypotheses $\left(H_{1}\right)$ and $\left(H_{2}\right)$. Then (1.1) is nonoscizlatory. 
Proof. We consider a sequence $\left\{y_{k}(t), k=0,1,2, \ldots\right\}$ of functions defined as follows:

$$
y_{0}(t)=\exp \left[-\left(\sigma_{0}\right)^{1 / n} t\right] ; \quad t \geq-\tau, \tau=\sup _{1 \leqq j \leqq n} \tau j(t), \quad t \geqq 0 .
$$

$$
y_{k+1}(t)=\left\{\begin{array}{l}
\exp \left[-\left(\sigma_{0}\right)^{1 / n} t\right] ; t \in[-\tau, 0] \\
\sum_{j=1}^{m} \int_{t}^{\infty} \frac{(s-t)^{n-1}}{(n-1) !} a_{j}(s) y_{k}\left(s-\tau_{j}(s)\right) d s, t>0 .
\end{array}\right.
$$

A direct verification leads to

$$
\begin{array}{rlrl}
y_{1}(t) & \leqq \sum_{j=1}^{m} \int_{t}^{\infty} \frac{(s-t)^{n-1}}{(n-1) !} a_{j}^{*} \exp \left[-\left(\sigma_{0}\right)^{1 / n}\left(s-\tau_{j}(s)\right)\right] d s \\
& \leqq y_{0}(t) ; t>0 & t>0
\end{array}
$$

from which one can derive that

$$
y_{2}(t) \leqq y_{1}(t) ; \quad t>0 .
$$

In a similar way one derives

$$
\begin{aligned}
y_{0}(t) \geqq y_{1}(t) \geqq \ldots \geqq y_{k}(t) \geqq y_{k+1}(t) \geqq \ldots & \geqq 0 \\
& t>0 .
\end{aligned}
$$

It will follow from (2.9) that the following limit exists in a pointwise sense;

$$
\lim _{k \rightarrow \infty} y_{k}(t)= \begin{cases}y^{*}(t) & \text { (say) } \text { for } t>0 \\ y_{0}(t) & \text { for } t \in[-\tau, 0] .\end{cases}
$$

By the Lebesgue's convergence theorem it follows from (2.10) that

$$
y^{*}(t)=\sum_{j=1}^{m} \int_{t}^{\infty}{\frac{(s-t)^{n-1}}{(n-1) !}}^{n}(s) y^{*}\left(s-\tau_{j}(s)\right) d s .
$$

The positivity of $y^{*}$ on $[0, \infty)$ follows from that of (2.11). Since $y^{*}$ in (2.11) is differentiable $n$-times for $t>0$ the result follows and the proof is complete.

\section{Nonoscillatory vector systems}

Let us now generalise our previous analysis to systems of the form 


$$
\frac{d^{n} X(t)}{d t^{n}}+(-1)^{n-1} A(t) X(t-\tau(t))=0, \quad t>0
$$

with the following assumptions:

$$
\begin{gathered}
\left(H_{3}\right) \quad X(t)=\left\{x_{1}(t), \ldots, x_{m}(t)\right\} \text { is an m-vector } \\
\left(H_{4}\right) \quad A(t)=\left\{a_{i j}(t)\right\} \text { is an } m \times m \text { matrix of bounded continuous } \\
\text { functions defined on }[0, \infty) \text { such that } \\
\quad 0 \leqq a_{i j}(t) \leqq l_{i j} \text { for all } t \geqq 0, \\
l_{i j} \text { being positive constants for } i, j=1,2, \ldots, n . \\
\left(H_{5}\right) \quad \tau(t) \text { is bounded and continuous for } t \geqq 0 \text { such that } \\
t-\tau(t) \geqq 0 \text { for } t \geqq 0 \\
0 \leqq \tau(t) \leqq \sigma \text { for } t \geqq 0 \text { and } \sigma>0 \text { is a constant. }
\end{gathered}
$$

Definitions of oscillatory and nonoscillatory systems of nonscalar equations have not become standard in the literature. In fact the literature concerned with oscillation and nonoscillation of vector matrix systems is quite scarce. We will adopt the following.

DEFINITION. The system (3.1) is said to nonoscillatory if at least one component of the vector $X(t)=\left\{x_{1}(t), \ldots, x_{n}(t)\right\}$ is nonoscillatory on $[0, \infty)$.

We note that the above is not the only way of generalising the notion of nonoscillation of scalax systems. For sone work on oscillation of linear systems we refer to [1].

We will first derive the following:

LEMMA 3.1. Let $B=\left(\ell_{i j}\right),(i, j=1,2, \ldots, m)$ be an $m \times m$ matrix of positive constants and let $\sigma$ be a positive constant. Then the autonomous linear system

$$
\frac{d^{n} Y(t)}{d t^{n}}+(-1)^{n-1} B Y(t-\sigma)=0
$$

is nonoscizlatory if

$$
\left(e^{n}{ }^{n} \alpha\right) / n^{n} \leqq 1
$$

where $\alpha$ is a positive Perron eigenvalue of $B$.

proof. A famous theorem of perron says that $B$ has a positive eigenvalue corresponding to which $B$ has a positive (componentwise) 


\section{K. Gopalsamy}

eigenvector. Let $\alpha_{1}, \alpha_{2}, \ldots, \alpha_{m}$ be the eigenvalues of $B$ of which at least one is positive; let it be denoted by $\alpha$. Let the corresponding eigenvector be $Z=\left(z_{1}, z_{2}, \ldots, z_{m}\right)$. The characteristic equation associated with $(3.2)$ is given by

$$
\operatorname{det} .\left[\lambda^{n} I+(-1)^{n-1} B e^{-\lambda \sigma}\right]=0
$$

( $I$ being the $m \times m$ identity matrix). Since we can rewrite (3.4) in the form

$$
\operatorname{det} .\left[(-\lambda)^{n} I-B e^{-\lambda \sigma}\right]=0
$$

we have from (3.5) that

$$
\operatorname{det} .\left[\mu^{n} I-B e^{\mu \sigma}\right]=0 \Leftrightarrow \prod_{j=1}^{m}\left[\mu^{n}-\alpha_{j} e^{\mu \sigma}\right]=0 \text {. }
$$

Let $\alpha$ denote a real eigenvalue from $\left(\alpha_{1}, \alpha_{2}, \ldots, \alpha_{m}\right)$. Then as in the case of our scalar analysis, it will follow from (3.3) and the equation

$$
\mu^{n}-\alpha e^{\mu \sigma}=0
$$

that (3.4) has a real negative root say $-\lambda^{*}$. It is now immediate that

$$
Y(t) \equiv 2 e^{-\lambda^{*} t}, \quad t \geq-\sigma
$$

is a nonoscillatory solution of (3.2) and the proof is complete.

We can now formulate our main result as follows:

THEOREM 3.1. Suppose the non-negative matrix $A$ in (3.1) is dominated (elementwise) by a positive constant matrix $B$ and the $t$-dependent dezay $\tau(t)$ is dominated by a positive constant $\sigma$ as in $\left(H_{3}\right)-\left(H_{5}\right)$. Let $\alpha$ be a real eigenvalue of $B$. If (3.3) holds then (3.1) is nonoscizlatory.

Proof. Proof is surprisingly simple and is quite similar to the scalar case; we define a sequence $\left\{X_{k}(t)\right\}$ of non-negative vectors as follows:

$$
\begin{gathered}
X_{0}(t) \equiv 2 e^{-\lambda^{*} t}, \quad t \geq-\tau \\
x_{k+1}(t)=\left\{\begin{array}{l}
2 e^{-\lambda^{*} t}, \quad t \in[-\tau, 0] \\
\int_{t}^{\infty} \frac{(s-t)^{n-1}}{(n-I) !} A(s) x_{k}(s-\tau(s)) d s, \quad t>0
\end{array}\right.
\end{gathered}
$$

where $Z$ is the positive eigenvector associated with the positive 
eigenvalue $\alpha$ of $B$. By componentwise comparison one can now show as in the scalar case that the sequence $\left\{x_{k}(t)\right\}$ has a positive (componentwise) pointwise limit say $X^{*}(t)$ which is a solution of (3.1); we will omit the details since they are exactly similar to the scalar case.

\section{Colarnents}

It is interesting to ask what can be said if (2.2) does not hold. We can say that when (2.2) is violated all bounded solutions of (2.3) will be oscillatory; to verify this assertion let us suppose

$$
\left[\sum_{j=1}^{m} a_{j}^{*}\left(\tau_{j}^{*}\right)^{n}\right] e^{n / n^{n}>1}
$$

and let (2.3) have a bounded nonoscillatory solution. Then (2.4) will have a real nonpositive root say $-\gamma$ with $\gamma \geq 0$ satisfying

$$
\gamma^{n}=\sum_{j=1}^{m} a_{j}^{*} \exp \left[\gamma \tau_{j}^{*}\right]
$$

Since $\gamma$ cannot be zero, we have $\gamma>0$ and hence (4.2) leads to

$$
1=\sum_{j=1}^{m} a_{j}^{*}\left(\tau_{j}^{*}\right)^{n}\left\{\exp \left[\gamma \tau_{j}^{*}\right]\right\} /\left(\tau_{j}^{*} \gamma\right)^{n} \geqq\left\{\sum_{j=1}^{m} a_{j}^{*}\left(\tau_{j}^{*}\right)^{n}\right\} e^{n / n^{n}}
$$

which contradicts (4.1). Thus without (4.1), (2.3) cannot have a bounded nonoscillatory solution. Similar remarks and arguments also apply to our condition (3.3) for the system (3.2) since we can consider a relevant factor of the characteristic equation in (3.6) (see also [1]). We mention some possible generalisations of our results; theorem (2.1) can be generalised to scalar systems of the form

$$
\frac{d^{n} x(t)}{d t^{n}}+(-1)^{n-1} \sum_{j=0}^{n-1}(-1)^{j} a_{j} \frac{d^{j} x\left(t-\tau_{j}\right)}{d t^{j}}=0
$$

with the assumption that $a_{j}, \tau_{j}$ are positive constants satisfying

$$
\left[\sum_{j=0}^{n-1} a_{j} \frac{(n+j)}{n}\left(\tau_{j} / n\right)^{n-j}\right] e^{n} \leqq 1 ;
$$

theorem 3.1 can be generalised for nonlinear systems of the form

$$
\frac{d^{n} x(t)}{d t^{n}}+(-1)^{n-1} A(t) f(t, X(t-\tau(t)))=0
$$

where $f$ is componentwise non-negative and nondecreasing with respect to 
its vector argument such that

$$
0 \leqq A(t) f(t, X(t)) \leqq B X(t) \text { for } X(t) \in \mathbb{R}_{m}^{+} \text {. }
$$

\section{References}

[1] K. Gopalsamy, "Oscillations in linear systems of differentialdifference equations", Bull. Austral. Math. Soc. 29 (1984), 377-384.

[2] A.G. Kartsatos, "Recent results on oscillation of solutions of forced and perturbed nonlinear differential equations of even order", 17-72 (Lecture notes in pure and applied mathematics, vol.28, Marcel Dekker, New York, 1977).

\footnotetext{
School of Mathematical Sciences,

Flinders University of South Australia,

Bedford Park,

S.A. 5042,

Australia.
} 